\title{
VALIDAÇÃO DA SINTAXE UNIFICADA PARA O CÁLCULO DOS ESCORES DOS INSTRUMENTOS WHOQOL
}

\author{
Bruno Pedroso, Faculdade de Educação Física, Universidade Estadual de Campinas - UNICAMP, \\ Campinas, São Paulo - Brasil \\ Luiz Alberto Pilatti, Universidade Tecnológica Federal do Paraná, Curitiba, Paraná - Brasil \\ Gustavo Luis Gutierrez, Faculdade de Educação Física, Universidade Estadual de Campinas - \\ UNICAMP, Campinas, São Paulo - Brasil \\ Celso Bilynkievycz dos Santos, Universidade Tecnológica Federal do Paraná, Curitiba, Paraná - \\ Brasil \\ Claudia Tânia Picinin, Universidade Tecnológica Federal do Paraná, Curitiba, Paraná - Brasil
}

\section{RESUMO}

O objetivo do presente trabalho é propor uma sintaxe unificada para a realização dos cálculos dos resultados dos instrumentos WHOQOL. As sintaxes originais dos instrumentos WHOQOL foram analisadas de forma a elencar os pontos divergentes entre estas. Foram selecionados critérios para a elaboração de uma sintaxe denominada "ideal", a qual abarca os aspectos que suprimem as fragilidades de individuais de cada instrumento WHOQOL. As sintaxes de cada instrumento foram reformuladas e testadas através de simulações com dados reais de aplicações dos instrumentos WHOQOL. Foi constatado que as sintaxes reformuladas, factualmente, suprimem as fragilidades presentes nas sintaxes dos instrumentos WHOQOL. Conclui-se que o rigor metodológico utilizado para a análise e teste das sintaxes preconizadas no presente estudo garante a fidedignidade das mesmas e que o objetivo de elaborar sintaxes que utilizam critérios ímpares para todos os instrumentos WHOQOL foi atingido.

PALAVRAS-CHAVE: Qualidade de vida, Sintaxes, Instrumentos WHOQOL.

\section{UNIFIED SYNTAX VALIDATION FOR CALCULATING SCORES OF WHOQOL INSTRUMENTS}

\begin{abstract}
The objective of this paper is to propose a unified syntax to accomplish the calculation of results from WHOQOL instruments. The original syntaxes of WHOQOL instruments were analyzed in a way to enlist the divergent points between them. Some criteria were selected for the development of a syntax called "ideal", which covers the aspects that suppress the individual weaknesses of each WHOQOL instrument. The syntax of each instrument was reformulated and tested through simulations with real data from applications of WHOQOL instruments. It was confirmed that the reformulated syntaxes, factually, suppress the weaknesses present in the syntaxes of WHOQOL instruments. It was concluded that the methodological strictness used to the analyze and test the syntaxes recommended in this study ensures the reliability of them, and the goal of developing syntaxes that use unique criteria for all WHOQOL instruments has been reached.
\end{abstract}

Key-Words: Quality of life, Syntaxes, WHOQOL instruments.

Conexões: revista da Faculdade de Educação Física da UNICAMP, Campinas, v. 9, n. 1, p. 130-156, jan./abr. 2011. ISBN: 1983-9030 


\section{INTRODUÇÃO}

O termo saúde foi, em 1946, definido pela Organização Mundial da Saúde (OMS) como "um completo estado de bem-estar físico, mental e social e não meramente a ausência de doença" (FLECK et al., 2000, p. 34). Ainda que date de mais de meio século a quebra do paradigma de que a saúde é o oposto de enfermo, a preocupação com a melhoria da saúde se intensificou em meados da década de 70 .

Mesmo havendo conformidade de opiniões sobre a importância de se avaliar a qualidade de vida, tal conceito apresenta dissensão. A multidimensionalidade do conceito de qualidade de vida abarca diferentes indicadores, propostos por autores que conformaram diferentes construções conceituais. Tal fato faz com que o peso atribuído aos indicadores promova a existência de conceitos distintos. ${ }^{1}$

O fato de não haver um consenso conceitual constitui um grande problema no desenvolvimento de instrumentos de avaliação da qualidade de vida, ao passo que não é possível afirmar com clareza quais elementos estes instrumentos estão mensurando. ${ }^{2}$

Partindo dessa premissa, o ponto de partida para a construção do instrumento de avaliação da qualidade de vida da OMS (World Health Organization Quality of Life - WHOQOL), em 1998, foi conceituar qualidade de vida. No conceito construído, qualidade de vida foi entendida como "a percepção do indivíduo de sua posição na vida no contexto da cultura e Conexões: revista da Faculdade de Educação Física da UNICAMP, Campinas, v. 9, n. 1, p. 130-156, jan./abr. 2011. ISBN: 1983-9030 
sistema de valores nos quais ele vive e em relação aos seus objetivos, expectativas, padrões e preocupações". ${ }^{3}$ (p. 199). Pautado nesse conceito, a OMS empenhou-se na construção dos instrumentos WHOQOL, que avaliam a qualidade de vida tanto de forma global (WHOQOL100, WHOQOL-bref, WHOQOL-SRPB) como também em função de grupos específicos (WHOQOL-HIV, WHOQOL-HIV-bref, WHOQOL-OLD).

Para a obtenção dos resultados das aplicações dos instrumentos WHOQOL, o Grupo WHOQOL recomenda o software estatístico Statistical Package for the Social Sciences (SPSS). Embora as sintaxes de todos os instrumentos WHOQOL tenham sido desenvolvidas no software SPSS, os procedimentos utilizados na sintaxe dos instrumentos WHOQOL não seguem uma padronização, diferindo-se em todos os instrumentos existentes. Os instrumentos WHOQOL são amplamente utilizados no âmbito da saúde pública. As fragilidades decorrentes dos distintos critérios presentes na sintaxe de cada instrumento WHOQOL, podem conduzir a resultados equivocados.

Nessa perspectiva, o presente estudo objetiva validar uma proposta unificada de sintaxes para os instrumentos WHOQOL. Como objetivo específico, tem-se a proposta de clarificar os procedimentos para o cálculo dos escores dos instrumentos WHOQOL, os quais estão dispostos implicitamente nos documentos publicados pelo Grupo WHOQOL. Justifica-se o intento em função da ampla utilização dos instrumentos em estudos dentro da área da saúde.

\section{O INSTRUMENTO PRECURSOR: WHOQOL-100}

O WHOQOL-100 é a versão completa do instrumento de avaliação da qualidade de vida da

OMS. É composto por 100 questões fechadas, que utilizam uma escala de respostas do tipo Likert, composta por cinco elementos, variando entre 1 e 5. Os resultados da aplicação do Conexões: revista da Faculdade de Educação Física da UNICAMP, Campinas, v. 9, n. 1, p. 130-156, jan./abr. 2011. ISBN: 1983-9030 
WHOQOL-100 são expressos através dos escores de cada faceta e domínio. A OMS aconselha a utilização do software estatístico SPSS para o cálculo dos resultados do WHOQOL-100.

Mesmo que a sintaxe SPSS para o WHOQOL-100 seja de domínio público, o Grupo WHOQOL apenas divulga as linhas de código da sintaxe, sem a preocupação de explicar os critérios para o cálculo dos escores dos domínios e facetas do WHOQOL-100. Na tentativa de suprimir essa omissão, neste estudo foram traduzidas as linhas de código da sintaxe SPSS, explicando quais são os procedimentos utilizados para o cálculo dos escores dos domínios e facetas do WHOQOL-100. Assim, pode-se afirmar que os resultados do WHOQOL-100 são calculados da seguinte forma:

- É verificado se todas as 100 questões foram preenchidas com valores entre 1 e 5 ;

- Invertem-se as 18 questões cuja escala de respostas é invertida;

- Os escores das facetas são calculados a partir da média aritmética simples das questões que compõem cada faceta, seguido de uma multiplicação por quatro. A multiplicação por quatro é utilizada para que, no caso de uma questão não ter sido respondida, o escore da faceta compense a nulidade da questão através do produto pelo número de questões válidas que a faceta deveria ter. Serão computadas somente as facetas que possuírem pelo menos três itens válidos;

- São calculados os escores dos domínios através da média aritmética simples entre os escores das facetas que compõem cada domínio. Nos domínios compostos por até cinco facetas, este será calculado somente se o número de facetas não calculadas não for igual ou superior a dois. Nos domínios Conexões: revista da Faculdade de Educação Física da UNICAMP, Campinas, v. 9, n. 1, p. 130-156, jan./abr. 2011. ISBN: 1983-9030 
compostos por mais de cinco facetas, este será calculado somente se o número de facetas não calculadas não for igual ou superior a três. No caso de facetas em escala invertida (todas as questões pertencentes à faceta possuem escala de respostas invertida), realiza-se a inversão dessa faceta para o prosseguimento do cálculo;

- Os escores dos domínios e facetas são convertidos para uma escala de 0 a 100

- Realiza-se uma contagem do total de itens respondidos por cada respondente. São computados no cálculo somente os respondentes que preencheram corretamente pelo menos 80 itens $(80 \%$ dos itens do instrumento).

Os resultados do WHOQOL-100 são expressos em uma escala variante entre 4 e 20 pontos. Tal escala se deve ao fato do cálculo do escore das facetas ser realizado através da multiplicação da média das questões que constituem cada faceta por quatro. Como cada domínio é calculado através da média aritmética simples das facetas que o compõem, os resultados são expressos na mesma escala das facetas.

\section{Conversão da escala de respostas das questões e facetas invertidas}

A conversão das questões é utilizada com o objetivo de padronizar todas as respostas do instrumento, de forma que a resposta mais positiva seja 5. Por conseguinte, a resposta mais negativa deve ser 1 . Desta forma, todas as questões de cada faceta são convertidas para uma mesma escala, onde o aumento gradativo da resposta equivale, na mesma proporção, ao aumento no positivismo do resultado da faceta.

Conexões: revista da Faculdade de Educação Física da UNICAMP, Campinas, v. 9, n. 1, p. 130-156, jan./abr. 2011. 
No caso de todas as quatro questões que constituem uma faceta sejam dispostas em escala invertida, essa mesma lógica é utilizada, mas somente no cálculo do domínio. Ou seja, o resultado dessas facetas é expresso na escala original, sem inversão. Entretanto, ao se calcular o escore dos domínios nos quais tais facetas estão inseridas, o escore dessas é convertido.

Para a conversão da escala de resposta das questões, o valor mínimo da questão de escala invertida deve ser substituído pelo valor máximo da questão de escala normal, assim como o valor máximo da questão de escala invertida deve ser substituído pelo valor mínimo da questão de escala normal. O mesmo deve ocorrer com os valores intermediários, seguindo essa mesma lógica. Assim, o único valor que não é alterado é o valor central, que tanto na escala normal, quanto na escala invertida, permanece o mesmo.

É preciso estar atento a esse fato, pois ao comparar os resultados entre as facetas, o escore de uma faceta de escala invertida não pode ser diretamente comparado ao escore de uma faceta de escala normal. Pode se observar no Quadro 1 os valores assumidos pelas respostas de questões cuja escala é invertida:

\section{Quadro 1 - Conversão de escala}

\begin{tabular}{lccccc}
\hline TIPO DE ESCALA & $\mathbf{0 \%}$ & $\mathbf{2 5 \%}$ & $\mathbf{5 0 \%}$ & $\mathbf{7 5 \%}$ & $\mathbf{1 0 0 \%}$ \\
\hline Normal & 1 & 2 & 3 & 4 & 5 \\
Invertida & 5 & 4 & 3 & 2 & 1 \\
\hline Fonte: Autoria própria & & & & &
\end{tabular}

O mesmo procedimento é utilizado na conversão de facetas invertidas. Após a conversão de uma faceta negativa, os valores "âncora" 4, 8, 12, 16 e 20 passam a assumir os valores 20, 16, 12, 8 e 4, respectivamente. Valores intermediários são convertidos na mesma proporção.

\section{Questões, domínios e facetas do WHOQOL-100}

Conexões: revista da Faculdade de Educação Física da UNICAMP, Campinas, v. 9, n. 1, p. 130-156, jan./abr. 2011. ISBN: 1983-9030 
O WHOQOL-100 é seccionado em 24 grupos de quatro questões cada, recebendo a denominação de "facetas". Por sua vez, o conjunto de facetas constitui um "domínio". Ao contrário da composição das facetas, os seis domínios do WHOQOL-100 não são constituídos pelo mesmo número de facetas, podendo variar de um até oito facetas.

As questões que compõem o WHOQOL-100 não estão dispostas no questionário seguindo uma seqüência lógica por domínio ou por faceta. A distribuição das facetas e domínios do WHOQOL-100 está relacionada no Quadro 2:

Quadro 2 - Domínios e facetas dos WHOQOL-100

\begin{tabular}{|c|c|}
\hline DOMÍNIOS & FACETAS \\
\hline \multirow{3}{*}{ Domínio I - Domínio físico } & 1. Dor e desconforto \\
\hline & 2. Energia e fadiga \\
\hline & 3. Sono e repouso \\
\hline \multirow{5}{*}{ Domínio II - Domínio psicológico } & 4. Sentimentos positivos \\
\hline & 5. Pensar, aprender, memória e concentração \\
\hline & 6. Auto-estima \\
\hline & 7. Imagem corporal e aparência \\
\hline & 8. Sentimentos negativos \\
\hline \multirow{4}{*}{ Domínio III - Nível de Independência } & 9. Mobilidade \\
\hline & 10. Atividades da vida cotidiana \\
\hline & 11. Dependência de medicação ou de tratamentos \\
\hline & 12. Capacidade de trabalho \\
\hline \multirow{3}{*}{ Domínio IV - Relações sociais } & 13. Relações pessoais \\
\hline & 14. Suporte (Apoio) social \\
\hline & 15. Atividade sexual \\
\hline \multirow{8}{*}{ Domínio V-Ambiente } & 16. Segurança física e proteção \\
\hline & 17. Ambiente no lar \\
\hline & 18. Recursos financeiros \\
\hline & 19. Cuidados de saúde e sociais: disponibilidade e qualidade \\
\hline & 20. Oportunidades de adquirir novas informações e habilidades \\
\hline & 21. Participação em, e oportunidades de recreação/lazer \\
\hline & 22. Ambiente físico: (poluição/ruído/trânsito/clima) \\
\hline & 23. Transporte \\
\hline $\begin{array}{l}\text { Domínio VI- Aspectos } \\
\text { espirituais/Religião/Crenças pessoais }\end{array}$ & 24. Espiritualidade/religião/crenças pessoais \\
\hline
\end{tabular}

Fonte: Grupo WHOQOL (1998)

Conexões: revista da Faculdade de Educação Física da UNICAMP, Campinas, v. 9, n. 1, p. 130-156, jan./abr. 2011. 
O WHOQOL-100 possui uma faceta que não está inserida em nenhum domínio, a faceta "Qualidade de vida global e percepção geral da saúde” (The WHOQOL Group, 1998a). Esta faceta aborda uma auto-avaliação da qualidade de vida, onde o respondente avalia a satisfação com a sua vida, saúde e qualidade de vida.

\section{DEMAIS INSTRUMENTOS WHOQOL}

\section{WHOQOL-bref}

Com o objetivo de disponibilizar um instrumento que demande menor tempo para preenchimento e com características psicométricas satisfatórias, o Grupo WHOQOL desenvolveu a versão abreviada do WHOQOL-100, o WHOQOL-bref (The WHOQOL Group, 1996).

O WHOQOL-bref é composto por 26 questões, sendo duas questões sobre a auto-avaliação da qualidade de vida e 24 questões representando cada uma das facetas do WHOQOL-100. Para a composição das questões do WHOQOL-bref foi selecionada a questão de cada faceta que apresentava a maior correlação com o escore médio de todas as facetas. ${ }^{4}$

Após a seleção das questões, foi realizada uma análise para verificar se estas, factualmente, representavam as facetas correspondentes. Em seis facetas, a questão selecionada foi substituída por outra questão da faceta correspondente, pois, sob o viés de estudiosos da área, havia outra questão que melhor definisse essas seis facetas. ${ }^{4}$ As facetas pertencentes ao domínio Nível de Independência foram incorporadas ao domínio Físico, assim como a faceta pertencente ao domínio Aspectos espirituais/Religião/Crenças pessoais foi incorporada ao domínio Psicológico. Assim, o WHOQOL-bref é composto por quatro domínios: Físico, 
Psicológico, Relações Sociais e Meio-Ambiente, perfazendo a configuração expressa no Quadro 3:

Quadro3 - Domínios e facetas do WHOQOL-bref

\begin{tabular}{|c|c|}
\hline DOMÍNIOS & FACETAS \\
\hline \multirow{7}{*}{ Domínio I - Domínio físico } & 1. Dor e desconforto \\
\hline & 2. Energia e fadiga \\
\hline & 3. Sono e repouso \\
\hline & 4. Mobilidade \\
\hline & 5. Atividades da vida cotidiana \\
\hline & 6. Dependência de medicação ou de tratamentos \\
\hline & 7. Capacidade de trabalho \\
\hline \multirow{6}{*}{ Domínio II - Domínio psicológico } & 8. Sentimentos positivos \\
\hline & 9. Pensar, aprender, memória e concentração \\
\hline & 10. Auto-estima \\
\hline & 11. Imagem corporal e aparência \\
\hline & 12. Sentimentos negativos \\
\hline & 13. Espiritualidade/religião/crenças pessoais \\
\hline \multirow{3}{*}{ Domínio III - Relações sociais } & 14. Relações pessoais \\
\hline & 15. Suporte (Apoio) social \\
\hline & 16. Atividade sexual \\
\hline \multirow{8}{*}{ Domínio IV- Meio-Ambiente } & 17. Segurança física e proteção \\
\hline & 18. Ambiente no lar \\
\hline & 19. Recursos financeiros \\
\hline & 20. Cuidados de saúde e sociais: disponibilidade e qualidade \\
\hline & 21. Oportunidades de adquirir novas informações e habilidades \\
\hline & 22. Participação em, e oportunidades de recreação/lazer \\
\hline & 23. Ambiente físico: (poluição/ruído/trânsito/clima) \\
\hline & 24. Transporte \\
\hline
\end{tabular}

Fonte: The WHOQOL Group (1998b)

O cálculo dos escores do WHOQOL-bref segue a mesma lógica do WHOQOL-100, exceto pelo cálculo dos escores das facetas. No WHOQOL-bref cada faceta é representada por uma única questão, e, por esse motivo, os escores das facetas não são calculados. ${ }^{5}$.

\section{WHOQO-HIV e WHOQOL-HIV-bref}

Com o objetivo de criar um instrumento de avaliação da qualidade de vida direcionado para portadores do HIV, pesquisadores da Joint United Nations Programme on HIV/AIDS (UNAIDS) e da WHO realizaram estudos em portadores do HIV em nove diferentes países. O

Conexões: revista da Faculdade de Educação Física da UNICAMP, Campinas, v. 9, n. 1, p. 130-156, jan./abr. 2011. ISBN: 1983-9030 
resultado desse estudo foi o instrumento WHOQOL-HIV, um módulo adicional específico para pessoas portadoras do HIV ou AIDS. ${ }^{6}$

O WHOQOL-HIV avalia a qualidade de vida a partir de seis domínios e 29 facetas. Os domínios e as facetas são as mesmas do WHOQOL-100, com o acréscimo de cinco facetas específicas para portadores do HIV. A faceta do WHOQOL-100 que avalia a qualidade de vida do ponto de vista do avaliado, não inserida em nenhum domínio, permanece no WHOQOL-HIV. As facetas específicas para os portadores do HIV, assim como as facetas do WHOQOL-100, são compostas por quatro questões. ${ }^{7}$ Frente a essa descrição, as facetas adicionais do WHOQOL-HIV se inserem nos domínios já existentes do WHOQOL-100, como pode ser observado no Quadro 4:

Quadro 4 - Domínios e facetas exclusivas do WHOQOL-HIV

\begin{tabular}{ll}
\hline \multicolumn{1}{c}{ DOMÍNIOS } & \multicolumn{1}{c}{ FACETAS } \\
\hline Domínio I - Físico & 50. Sintomas de PVHAs \\
Domínio IV - Relações sociais & 51. Inclusão social \\
Domínio VI - Aspectos & 52. Perdão e culpa \\
espirituais/Religião/Crenças pessoais & 53. Preocupações sobre o futuro \\
& 54. Morte e morrer \\
\hline Fonte: Adaptado de O'Connell et al.,2004
\end{tabular}

Fonte: Adaptado de O'Connell et al., 2004.

O cálculo dos resultados do WHOQOL-HIV é similar ao cálculo dos resultados WHOQOL100. No entanto, alguns critérios utilizados no WHOQOL-100 não foram herdados pelo WHOQOL-HIV. Os resultados do WHOQOL-HIV são calculados da seguinte forma:

- É verificado se todas as 120 questões foram preenchidas com valores entre 1 e 5;

- Invertem-se todas as questões cuja escala de respostas é invertida. Em se tratando de facetas em escala invertida, todas as questões pertencentes a estas facetas são invertidas individualmente;

Conexões: revista da Faculdade de Educação Física da UNICAMP, Campinas, v. 9, n. 1, p. 130-156, jan./abr. 2011. 
- Os escores das facetas são calculados a partir da soma das quatro questões pertencentes a cada faceta, seguido de uma divisão por quatro, sendo representados em uma escala de 1 a 5 ;

- Os escores dos domínios são calculados através da soma dos escores das "n" facetas que compõem cada domínio, dividido pelo número de facetas do domínio. O resultado é multiplicado por quatro, sendo representado em uma escala de 4 a 20.

Sob a mesma justificativa da elaboração do WHOQOL-bref, o Grupo WHOQOL desenvolveu uma versão abreviada do WHOQOL-HIV. O WHOQOL-HIV-bref é baseado no WHOQOLbref, da forma que cada faceta é representada por uma única questão. As 26 questões do WHOQOL-bref se repetem no WHOQOL-HIV-bref, sendo estas acrescidas de cinco questões que representam as facetas adicionais do WHOQOL-HIV. ${ }^{8}$ Ao contrário do que ocorre no WHOQOL-bref, as facetas pertencentes aos domínios nível de independência e Aspectos espirituais/Religião/Crenças pessoais não são incorporadas aos domínios Físico e Psicológico, assumindo, assim, a mesma configuração dos domínios do WHOQOL-HIV. O cálculo dos escores do WHOQOL-HIV-bref segue a mesma lógica presente no WHOQOL-bref.

\section{WHOQOL-OLD}

Com o intuito de adaptar o WHOQOL para a aplicação em adultos idosos, o Grupo WHOQOL desenvolveu um módulo adicional aos instrumentos WHOQOL-100 e WHOQOLbref, denominado WHOQOL-OLD. Ao contrário do WHOQOL-HIV, o WHOQOL-OLD não suplementa o WHOQOL-100 ou o WHOQOL-bref. O WHOQOL-OLD é um módulo complementar, mas que deve ser aplicado em conjunto com o WHOQOL-100 ou o 
WHOQOL-bref. $^{9}$ O WHOQOL-OLD, conforme ilustrado no Quadro 5, é composto por 24 questões seccionadas em seis facetas:

Quadro 5: Facetas do WHOQOL-OLD

\begin{tabular}{cl}
\hline \multicolumn{1}{c}{ DOMÍNIOS } & \multicolumn{1}{c}{ FACETAS } \\
\hline & 25. Habilidades sensoriais \\
& 26. Autonomia \\
Domínio VII - Envelhecimento & 27. Atividades passadas, presentes e futuras \\
& 28. Participação social \\
& 29. Morte e morrer \\
30. Intimidade
\end{tabular}

Fonte: Power et al. , 2005.

O cálculo dos escores do WHOQOL-OLD possui algumas particularidades com relação aos demais instrumentos WHOQOL, sendo calculado a partir da seguinte lógica:

- É verificado se todas as 24 questões foram preenchidas com valores entre 1 e 5;

- Invertem-se todas as questões cuja escala de respostas é invertida. Em se tratando de facetas em escala invertida, todas as questões pertencentes a estas facetas são invertidas individualmente;

- Os escores das facetas são calculados de três maneiras diferentes. O escore bruto é calculado a partir da soma de todas as questões pertencentes a cada uma das facetas, retornando um resultado variante entre 4 e 20 . O escore padronizado é calculado por meio da divisão do escore bruto da faceta pelo número de questões da faceta (quatro), retornando um resultado variante entre 1 e 5. O escore transformado é calculado a partir da conversão do escore bruto para uma escala de 0 a 100 ;

- O escore total do WHOQOL-OLD é calculado, também, de três formas. Através da soma das 24 questões do instrumento é obtido o escore bruto, 
variante entre 24 e 120. A divisão do escore bruto pelo número de questões do instrumento (24) representa o escore padronizado, variante entre 1 e 5 . A conversão do escore bruto para uma escala de 0 a 100 representa o escore transformado.

\section{WHOQOL SRPB}

Ao se perceber que os aspectos espirituais, a religião e as crenças pessoais apresentavam grande importância na avaliação da qualidade de vida em todo o mundo, teve a progênie o estudo do WHOQOL-SRPB. De acordo com Fleck e Skevington, ${ }^{10}$ o WHOQOL-SRPB tratase de um "estudo transcultural para desenvolver uma medida que avalie de que forma espiritualidade, religião e crenças pessoais (SRPB, sigla em inglês) estão relacionadas à qualidade de vida $(\mathrm{QV})$ na saúde e na assistência à saúde".

Em estudos posteriores ao desenvolvimento do WHOQOL-100 e WHOQOL-bref, foi constatado que o domínio Aspectos espirituais/Religião/Crenças pessoais nestes instrumentos era conceitualmente e empiricamente insuficiente. Frente a essa fragilidade, o Grupo WHOQOL pautou-se no desenvolvimento de um estudo para avaliar a forma com a qual o domínio em exame está relacionado com a qualidade de vida. ${ }^{11}$

O WHOQOL-SRPB não é um instrumento para avaliar a espiritualidade, mas sim, um instrumento que contempla, de forma ampliada, o constructo Aspectos espirituais/Religião/Crenças pessoais, representado no WHOQOL-100 e WHOQOL-bref de forma reduzida. ${ }^{11}$

Conexões: revista da Faculdade de Educação Física da UNICAMP, Campinas, v. 9, n. 1, p. 130-156, jan./abr. 2011. 
Nessa perspectiva, o WHOQOL-SRPB suplementa o WHOQOL-100 com oito facetas adicionais ao domínio Aspectos espirituais/Religião/Crenças pessoais. Os demais domínios do WHOQOL-100 permanecem inalterados. As facetas adicionais do WHOQOL-SRPB podem ser observadas no Quadro 6:

Quadro 6: Facetas adicionais do WHOQOL-SRPB

\begin{tabular}{ll}
\hline \multicolumn{1}{c}{ DOMÍNIOS } & \multicolumn{1}{c}{ FACETAS } \\
\hline & S1. Conexão com ser ou força espiritual \\
& S2. Sentido da vida \\
& S3. Admiração \\
Domínio VI - Aspectos & S4. Totalidade/integração \\
espirituais/Religião/Crenças pessoais & S5. Força interior \\
& S6. Paz interior/serenidade/harmonia \\
& S7. Esperança/otimismo \\
& S8. Fé \\
\hline
\end{tabular}

Fonte: The WHOQOL-SRPB Group (2006)

O cálculo dos escores dos domínios e facetas do WHOQOL-SRPB segue a mesma lógica presente no WHOQOL-HIV, de forma que os escores das facetas são apresentados em uma escala de 1 a 5 e os escores dos domínios são apresentados em uma escala de 4 a $20 .{ }^{11}$

\section{METODOLOGIA}

As sintaxes foram interpretadas, transcritas textualmente e analisadas. As principais disparidades entre os instrumentos foram identificadas. Os instrumentos destoavam no que diz respeito a oito critérios:

- Conversão das questões cuja escala de respostas é invertida;

- Procedimento para o cálculo dos escores das facetas;

- Escala na qual é exibido os escores das facetas;

- Mínimo de questões válidas para o cálculo da faceta;

- Procedimento para o cálculo dos escores dos domínios;

- Escala na qual é exibido os escores dos domínios;

Conexões: revista da Faculdade de Educação Física da UNICAMP, Campinas, v. 9, n. 1, p. 130-156, jan./abr. 2011. ISBN: 1983-9030 
- Mínimo de facetas válidas para o cálculo do domínio;

- Número mínimo de questões respondidas para que o respondente não seja excluído da amostragem.

As semelhanças e divergências em que concerne cada um dos critérios levantados são abordadas na seqüência.

\section{Conversão das questões cuja escala de respostas é invertida}

- WHOQOL-100: as questões que apresentam escala de respostas invertidas são convertidas. As questões inseridas em facetas onde todas as questões que a compõe são invertidas, não são convertidas. Nesse caso, o escore da faceta é invertido no cálculo do domínio ao qual esta pertence.

- WHOQOL-HIV: todas as questões que apresentam escala de respostas invertida são convertidas. As questões pertencentes à facetas invertidas são convertidas individualmente.

- WHOQOL-OLD: todas as questões que apresentam escala de respostas invertida são convertidas. As questões pertencentes à facetas invertidas são convertidas individualmente.

- WHOQOL-SRPB: todas as questões que apresentam escala de respostas invertida são convertidas. As questões pertencentes à facetas invertidas são convertidas individualmente.

- WHOQOL-bref: todas as questões que apresentam escala de respostas invertida são convertidas.

- WHOQOL-HIV-bref: todas as questões que apresentam escala de respostas invertida são convertidas.

Conexões: revista da Faculdade de Educação Física da UNICAMP, Campinas, v. 9, n. 1, p. 130-156, jan./abr. 2011. 


\section{Procedimento para o cálculo dos escores das facetas}

- WHOQOL-100: os escores das facetas são calculados a partir da média aritmética simples das questões que compõem cada faceta, seguido de uma multiplicação por quatro.

- WHOQOL-HIV: os escores das facetas são calculados a partir da soma das quatro questões pertencentes a cada faceta, seguido de uma divisão por quatro. Isso significa que o escore da faceta representa a média desta somente se todas as quatro questões forem corretamente preenchidas.

- WHOQOL-OLD: os escores das facetas são calculados a partir de três fórmulas diferentes, o que garante a exibição dos resultados em três escalas distintas. São calculados a partir da soma das quatro questões pertencentes a cada faceta; calculados a partir da média das quatro questões pertencentes a cada faceta; partindo do escore calculado através da média das questões, estes são convertidos para uma escala centesimal.

- WHOQOL-SRPB: os escores das facetas são calculados a partir da soma das quatro questões pertencentes a cada faceta, seguido de uma divisão por quatro. Isso significa que o escore da faceta representa a média desta somente se todas as quatro questões forem corretamente preenchidas.

- WHOQOL-bref: cada faceta é representada por uma única questão, e, por esse motivo, os escores das facetas não são calculados.

- WHOQOL-HIV-bref: cada faceta é representada por uma única questão, e, por esse motivo, os escores das facetas não são calculados. 


\section{Escala na qual é exibido os escores das facetas}

- WHOQOL-100: os escores das facetas são exibidos em uma escala de 4 a 20, e, também, em uma escala de 0 a 100.

- WHOQOL-HIV: os escores das facetas são exibidos em uma escala de 1 a 5.

- WHOQOL-OLD: os escores das facetas são exibidos em uma escala de 4 a 20, de 1 a 5 e de 0 a 100.

- WHOQOL-SRPB: os escores das facetas são exibidos em uma escala de 1 a 5.

- WHOQOL-bref: os escores das facetas não são calculados.

- WHOQOL-HIV-bref: os escores das facetas não são calculados.

\section{Mínimo de questões válidas para o cálculo da faceta}

- WHOQOL-100: serão computadas somente as facetas que possuírem pelo menos três itens válidos.

- WHOQOL-HIV: o escore da faceta é calculado desde que esta possua um item válido.

- WHOQOL-OLD: serão computadas somente as facetas que possuírem todos os quatro itens válidos.

- WHOQOL-SRPB: o escore da faceta é calculado desde que esta possua um item válido.

- WHOQOL-bref: escores não calculados.

- WHOQOL-HIV-bref: escores não calculados. 


\section{Procedimento para o cálculo dos escores dos domínios}

-WHOQOL-100: os escores dos domínios são calculados através da média aritmética simples entre os escores das facetas que compõem cada domínio.

-WHOQOL-HIV: os escores dos domínios são calculados através da soma dos escores das "n” facetas que compõem cada domínio, dividido pelo número de facetas do domínio. O resultado é multiplicado por quatro. O escore do domínio representa a média desse, unicamente, quando todas as facetas pertencentes a este são pontuadas.

-WHOQOL-OLD: o escore do domínio (ou escore total do instrumento) é calculado a partir de três fórmulas diferentes, o que garante a exibição dos resultados em três escalas distintas. É calculado a partir da soma das 24 questões pertencentes a cada faceta; calculado a partir da média das 24 questões pertencentes a cada faceta; partindo do escore calculado através da média das questões, estes são convertidos para uma escala de centesimal.

-WHOQOL-SRPB: os escores dos domínios são calculados através da soma dos escores das "n" facetas que compõem cada domínio, dividido pelo número de facetas do domínio. O resultado é multiplicado por quatro. O escore do domínio representa a média desse, unicamente, quando todas as facetas pertencentes a este são pontuadas.

-WHOQOL-bref: os escores dos domínios são calculados através da média aritmética simples entre os escores das questões (facetas) que compõem cada domínio, seguido de uma multiplicação por quatro.

-WHOQOL-HIV-bref: os escores dos domínios são calculados através da soma dos escores das "n" facetas que compõem cada domínio, dividido pelo 
número de facetas do domínio. O resultado é multiplicado por quatro. O escore do domínio representa a média desse, unicamente, quando todas as facetas pertencentes a este são pontuadas.

\section{Escala na qual é exibido os escores dos domínios}

- WHOQOL-100: os escores dos domínios são expressos em uma escala de 4 a 20, e, também, em uma escala de 0 a 100.

- WHOQOL-HIV: os escores dos domínios são expressos em uma escala de 4 a 20.

- WHOQOL-OLD: o escore do domínio é exibido em escalas de 24 a 120, 1 a 5 e 0 a 100.

- WHOQOL-SRPB: os escores dos domínios são expressos em uma escala de 4 a 20.

- WHOQOL-bref: os escores dos domínios são expressos em uma escala de 4 a 20, e, também, em uma escala de 0 a 100.

- WHOQOL-HIV-bref: os escores dos domínios são expressos em uma escala de 4 a 20.

\section{Mínimo de facetas válidas para o cálculo do domínio}

- WHOQOL-100: nos domínios compostos por até cinco facetas, este será calculado somente se o número de facetas não calculadas não for igual ou superior a dois. Nos domínios compostos por mais de cinco facetas, este será calculado somente se o número de facetas não calculadas não for igual ou superior a três.

Conexões: revista da Faculdade de Educação Física da UNICAMP, Campinas, v. 9, n. 1, p. 130-156, jan./abr. 2011. 
- WHOQOL-HIV: o escore do domínio é calculado desde que este possua uma faceta cujo escore não seja nulo.

- WHOQOL-OLD: o escore do domínio é calculado somente se as todas as 24 questões do instrumento são pontuadas.

- WHOQOL-SRPB: o escore do domínio é calculado desde que este possua uma faceta cujo escore não seja nulo.

- WHOQOL-bref: nos domínios compostos por até sete questões (facetas), este será calculado somente se o número de questões (facetas) não calculadas não for igual ou superior a dois. Nos domínios compostos por mais de sete questões (facetas), este será calculado somente se o número de questões (facetas) não calculadas não for igual ou superior a três.

- WHOQOL-HIV-bref: o escore do domínio é calculado desde que este possua uma faceta cujo escore não seja nulo.

Número mínimo de questões respondidas para que o respondente não seja excluído da amostragem

- WHOQOL-100: realiza-se uma contagem do total de itens respondidos por cada respondente. São computados no cálculo somente os respondentes que preencheram corretamente pelo menos 80 itens $(80 \%$ dos itens do instrumento).

- WHOQOL-HIV: não há contagem de itens respondidos. Mesmo que o número de itens respondidos seja inferior a $80 \%$, suas respostas são computadas nos cálculos dos resultados.

Conexões: revista da Faculdade de Educação Física da UNICAMP, Campinas, v. 9, n. 1, p. 130-156, jan./abr. 2011. 
- WHOQOL-OLD: não há contagem de itens respondidos. Ainda que as facetas sejam calculadas somente se todos os itens forem válidos e o domínio seja calculado somente de $100 \%$ das questões do instrumento sejam válidas, caso um respondente responda somente quatro questões da mesma faceta, totalizando $16,67 \%$ do total de questões, o escore desta faceta será calculado e o respondente não será descartado.

- WHOQOL-SRPB: não há contagem de itens respondidos. Mesmo que o número de itens respondidos seja inferior a $80 \%$, suas respostas são computadas nos cálculos dos resultados.

- WHOQOL-bref: realiza-se uma contagem do total de itens respondidos por cada respondente. São computados no cálculo somente os respondentes que preencheram corretamente pelo menos 21 itens $(80 \%$ dos itens do instrumento).

- WHOQOL-HIV-bref: não há contagem de itens respondidos. Mesmo que o número de itens respondidos seja inferior a $80 \%$, suas respostas são computadas nos cálculos dos resultados.

\section{Procedimentos utilizados na construção das sintaxes}

Concluída a analogia entre as sintaxes dos instrumentos WHOQOL, foram elencados os pontos positivos e negativos de cada instrumento. Frente às características levantadas, estas foram criteriosamente analisadas e debatidas, objetivando a seleção das características que ofereciam maior fidedignidade a cada instrumento levando em consideração cada um dos oito critérios onde foi constatado divergências entre os instrumentos.

Conexões: revista da Faculdade de Educação Física da UNICAMP, Campinas, v. 9, n. 1, p. 130-156, jan./abr. 2011. 
Finalizada a seleção das características que comporiam a "sintaxe ideal", as sintaxes de todos os instrumentos WHOQOL foram reformuladas. A reformulação das sintaxes consistiu em adequar todos os critérios da sintaxe original que divergiam dos critérios da "sintaxe ideal". Nessa perspectiva, todos os instrumentos WHOQOL passam a adotar as mesmas características entre si.

As sintaxes foram analisadas e testadas no software SPSS por pesquisadores curso de Mestrado em Engenharia de Produção e do curso de graduação em Análise e Desenvolvimento de Sistemas da UTFPR - Campus Ponta Grossa. Todos os erros constatados foram devidamente corrigidos.

\section{RESULTADOS}

O debate referente a analise das sintaxes dos instrumentos WHOQOL norteou a implementação de uma sintaxe denominada "ideal” para todos os instrumentos em exame. Os critérios selecionados para compor a "sintaxe ideal" foram:

- Conversão das questões cuja escala de respostas é invertida: todas as questões que apresentam escala de respostas invertida são convertidas. As questões pertencentes à facetas invertidas são convertidas individualmente.

- Escala na qual é exibido os escores dos facetas: os escores das facetas são exibidos em uma escala de 4 a 20, e, também, em uma escala de 0 a 100.

- Procedimento para o cálculo dos escores dos domínios: procedimento para o cálculo dos escores das facetas: os escores das facetas são calculados a partir da média aritmética simples das questões que compõem cada faceta, seguido de uma 
multiplicação por quatro. No caso das versões abreviadas, o escore da questão que compõe cada faceta é multiplicado por quatro.

- Mínimo de questões válidas para o cálculo da faceta: serão computadas somente as facetas que possuírem pelo menos três itens válidos. No caso das versões abreviadas do WHOQOL, caso a questão correspondente a determinada faceta tenha sido pontuada, o escore de tal faceta é calculado.

- Procedimento para o cálculo dos escores dos domínios: os escores dos domínios são calculados através da média aritmética simples entre os escores das facetas que compõem cada domínio.

- Escala na qual é exibido os escores dos domínios: os escores dos domínios são expressos em uma escala de 4 a 20, e, também, em uma escala de 0 a 100.

- Mínimo de facetas válidas para o cálculo do domínio: nos domínios compostos por até sete facetas, este será calculado somente se o número de facetas não calculadas não for igual ou superior a dois. Nos domínios compostos por mais de sete facetas, este será calculado somente se o número de facetas não calculadas não for igual ou superior a três.

- Número mínimo de questões respondidas para que o respondente não seja excluído da amostragem: realiza-se uma contagem do total de itens respondidos por cada respondente. São computados no cálculo somente os respondentes que preencheram corretamente pelo menos $80 \%$ dos itens do instrumento.

As sintaxes reformuladas propostas no presente trabalho realizam todos os cálculos propostos pelo Grupo WHOQOL, sendo que o procedimento para a utilização destas é exatamente o mesmo utilizado nas sintaxes originais. Algumas funcionalidades adicionais, julgadas pelos 
pesquisadores como relevantes e que eram ausentes nas sintaxes originais, foram acrescentadas. No que concerne às funcionalidades adicionais, foi acrescentado o cálculo dos escores das facetas no WHOQOL-bref e WHOQOL-HIV-bref, anteriormente inexistente em ambos os instrumentos mencionados.

A utilização de uma equipe multidisciplinar e de critérios rígidos na etapa de testes e validação das sintaxes reduz a possibilidade de incidência de erros nas sintaxes propostas. Para a validação das sintaxes, foram realizadas simulações com dados reais de aplicações de cada um dos instrumentos WHOQOL. Em se tratando que os resultados retornados pelas sintaxes reformuladas seriam diferentes dos retornados pelas sintaxes originais, o cálculo dos resultados das sintaxes reformuladas foi, com base nos critérios pré-estabelecidos pelos pesquisadores, calculado manualmente, a fim de comparação. Os resultados retornados pelo cálculo manual e pelo SPSS foram exatamente os mesmos, garantindo assim a fidedignidade das sintaxes objeto de estudo deste trabalho.

Os requisitos para a utilização das presentes ferramentas são: computador ou notebook com o software SPSS instalado. O download de todas as sintaxes pode ser realizado através da URL: http://www.brunopedroso.com.br/whoqol_uni.html.

\section{CONSIDERAÇÕES FINAIS}

Os instrumentos WHOQOL são os instrumentos de avaliação da qualidade de vida mais utilizados no mundo. Mesmo sendo amplamente difundidos, a dificuldade de interpretação de sua sintaxe e a existência de sintaxes bastante divergentes para o cálculo dos resultados dos instrumentos em exame, constituem fatores limitantes para sua utilização.

Conexões: revista da Faculdade de Educação Física da UNICAMP, Campinas, v. 9, n. 1, p. 130-156, jan./abr. 2011. ISBN: 1983-9030 
Almejando a supressão dessas limitações, o presente estudo transcreveu textualmente a sintaxe SPSS, detalhando todos os passos utilizados para a obtenção dos resultados dos instrumentos WHOQOL. A partir da análise de todas as sintaxes propostas pelo Grupo WHOQOL, foi, também, proposta uma sintaxe unificada para todos os instrumentos WHOQOL. A unificação das sintaxes consistiu na seleção dos critérios que, depois de debatidos, houve a constatação de que proporcionavam maior fidedignidade aos instrumentos analisados. Frente aos critérios selecionados, foi construída uma sintaxe "ideal", a qual foi adaptada para todos os instrumentos WHOQOL.

As sintaxes foram criteriosamente analisadas e testadas por pesquisadores do Mestrado em Engenharia de Produção e do curso de graduação em Tecnologia em Análise e Desenvolvimento de Sistemas da UTFPR - Campus Ponta Grossa. Todos os erros constatados foram devidamente corrigidos. Tendo em vista a impossibilidade de comparação dos resultados obtidos através da sintaxe reformulada e a sintaxe original de cada instrumento, os cálculos das sintaxes reformuladas foram realizados manualmente e comparados com suas respectivas sintaxes executadas no SPSS. Os resultados obtidos foram idênticos em ambas as situações.

A tentativa de desmistificação das sintaxes e criação de uma sintaxe unificada para todos os instrumentos WHOQOL foi alcançada. Na perspectiva apresentada, conclui-se que as reformulações das sintaxes propostas no presente estudo consistem na adoção de critérios ímpares para todos os instrumentos WHOQOL. A seleção dos critérios a ser implementados nas sintaxes reformuladas se compôs de uma detalhada análise, com o intuito de suprimir as fragilidades existentes em cada um dos instrumentos examinados. Foram, também, acrescidas funcionalidades adicionais ausentes nas sintaxes originais das versões abreviadas dos Conexões: revista da Faculdade de Educação Física da UNICAMP, Campinas, v. 9, n. 1, p. 130-156, jan./abr. 2011. ISBN: 1983-9030 
instrumentos WHOQOL. Por fim, cabe ressaltar que o rigor metodológico presente na proposta garante a fidedignidade das sintaxes disponibilizadas neste estudo.

\section{REFERÊNCIAS}

${ }^{1}$ FLECK, M. P. A. O instrumento de avaliação de qualidade de vida da Organização Mundial da Saúde (WHOQOL-100): características e perspectivas. Ciência e Saúde Coletiva. v. 5, n.1, p. 33-38, 2000.

${ }^{2}$ FLECK, M. P. A. et al. Problemas conceituais em qualidade de vida. In: FLECK, M. P. A. et al. A avaliação de qualidade de vida: guia para profissionais da saúde. Porto Alegre: Artmed, 2008.

${ }^{3}$ FLECK, M. P. A. et al. Aplicação da versão em português do instrumento de avaliação de qualidade de vida da Organização Mundial da Saúde (WHOQOL-100). Revista de Saúde Pública. v. 33, n. 2, p. 198-205, 1999a.

${ }^{4}$ The WHOQOL Group. Development of the World Health Organization WHOQOL-BREF Quality of Life Assessment. Psychological Medicine. v. 28, n. 3, p. 551-558, 1998 b.

${ }^{5}$ The WHOQOL Group. WHOQOL-bref: introduction, administration, scoring and generic version of assessment. Geneva: 1996.

${ }^{6}$ WHO Field Centre for the Study of Quality of Life of Bath. About the WHO Field Centre for the Study of Quality of Life. Disponível em: <http://www.bath.ac.uk/whoqol/about.cfm>. Acesso em: 13 abr. 2008.

Conexões: revista da Faculdade de Educação Física da UNICAMP, Campinas, v. 9, n. 1, p. 130-156, jan./abr. 2011. ISBN: 1983-9030 
${ }^{7}$ O'CONNELL, K. et al. WHOQOL-HIV for quality of life assessment among people living with HIV and AIDS: results from a field test. AIDS Care. v. 16, n. 7, p. 882-889, 2004.

${ }^{8}$ The WHOQOL-HIV Group. WHOQOL-HIV Instrument Users Manual. Geneva: 2002.

${ }^{9}$ POWER, M. et al. Development of the WHOQOL-Old module. Quality of Life Research. v. 14, n. 10, p. 2197-2214, 2005.

${ }^{10}$ FLECK, M. P. A.; SKEVINGTON, S. Explicando o significado do WHOQOL-SRPB. Revista de Psiquiatria Clínica. v. 34, Suplemento 1, p. 146-149, 2007.

${ }^{11}$ The WHOQOL-SRPB Group. A cross-cultural study of spirituality, religion, and personal beliefs as components of quality of life. Social Science and Medicine. v. 62, n. 6, p. 14861497, 2006. 